\title{
Next-generation Sequencing in the Clinical Decision Making in Hypertrophic Cardiomyopathy
}

Viktor J Horvath ${ }^{1^{*}}$, Kristof Arvai ${ }^{2}$, Janos P Kosa ${ }^{1}$, Bernadett Balla ${ }^{2}$, Balint Tobias ${ }^{2}$, Gyongyi Kirschner ${ }^{1}$, Zsuzsanna Putz ${ }^{1}$, Zsolt Nagy ${ }^{1}$, Istvan Takacs ${ }^{1}$, Laszlo Kornyei $^{3}$, Hajnalka Vago ${ }^{4}$, Attila Toth $^{4}$, Istvan Liko ${ }^{5}$, Gyorgy Fekete ${ }^{6}$, Bela Merkely ${ }^{4}$ and Peter Lakatos ${ }^{1}$

${ }_{11}^{1}$ st Department of Medicine, Semmelweis Universty, Budapest, Hungary

${ }^{2}$ PentaCore Laboratory, Budapest, Hungary

${ }^{3}$ Gottsegen György National Institute of Cardiology, Budapest, Hungary

${ }^{4}$ Heart and Cardiovascular Center, Semmelweis University, Budapest, Hungary

5"Lendület" Research Group of Inherited Endocrine Tumors, Hungarian Academy of Science, Budapest, Hungary

${ }^{6} 2$ nd Department of Pediatrics, Semmelweis University, Budapest, Hungary

*Corresponding author: Viktor J Horvath, 1st Department of Medicine, Semmelweis University, Budapest, Hungary, Tel: +0036206663281; Fax: +003613130250; Email: horvathjviktor@gmail.com

Rec date: Apr 10, 2017; Acc date: Apr 28, 2017; Pub date: May 02, 2017

Copyright: (c) 2017 Horvath VJ, et al. This is an open-access article distributed under the terms of the creative commons attribution license, which permits unrestricted use, distribution, and reproduction in any medium, provided the original author and source are credited.

\begin{abstract}
Next generation sequencing (NGS) is becoming a valuable tool in clinical decisions. Here, we discuss the case of a family (2 parents with 3 children) with hypertrophic cardiomyopathy where we applied a NGS method developed by us to determine the genetic background of the disease. When the youngest sister underwent sudden cardiac arrest and successful reanimation, her genome was tested with this approach and two disease-causing heterozygous mutations in the MYBPC3 gene (p.R495Q and p.S593fs*11) were identified. After this, all of the family members were screened targeting these two mutations. The mother carried the frameshift mutation (p.S593fs $\left.{ }^{*} 11\right)$ while the father's genome contained the point mutation ( $p . R 495 Q)$. All the children were compound heterozygous. Information collected from our genetic testing panel helped to make the decision of implanting ICD that is associated potentially severe complications in children. This case further reinforces that a full-scale, cost-effective NGS method can be utilized to supplement diagnostic and therapeutic processes of hypertrophic cardiomyopathy in clinical practice.
\end{abstract}

Keywords: Hypertrophic cardiomyopathy; Next generation sequencing; Frameshift mutation; Genome; Clinical practice

\section{Introduction}

Next generation sequencing (NGS) techniques are cost effective genetic tools with prominent performance [1]. Their widening access makes this approach a valuable tool for every laboratory either for research or clinical diagnosis. The gold standard technique was described [2], however, the price and time requirement of the technique hampered its fast gaining ground. Since next generation sequencing creates signals during the synthesis without the irreversible termination of the DNA strand in multiple wells at the same time, it could mean millions of DNA strands sequenced at once, which gives abundant information in much less time. Pyrosequencing, fluorescent detection of dNTP incorporation and semi-conductor sequencing technology are three approaches for gathering reliable information which can even help clinicians for making difficult therapeutical decisions. Approaching a clinical question with a help of semiconductor Ion Torrent has already been utilized for diagnosing some clinical entities and the method is able to directly perform nonoptical DNA sequencing of different genomes $[3,4]$.

In this study, our aim was to work out a NGS method utilizing the IonTorrent PGM (ThermoFisher Scientific, Waltham, MA, USA) in diagnostic settings for cardiologic diseases with known genetic background. Until recently, more than 40 cardiovascular disorders have been described as a consequence of a single gene defect [5].
Hypertrophic cardiomyopathy (HCM) is the most common cardiovascular disorder with genetic background occurring with a frequency of 1 in 500 adult people in a general population [6] and with an incidence of $0.3-0.5$ per 100,000 among children [7]. More than 400 known mutations in at least 13 , mostly cardiac sarcomeric protein genes have been related to the development of HCM [8]. The most frequently involved genes are cardiac $\beta$-myosin heavy chain ( $\beta \mathrm{MHC}$; $35 \%)$ and cardiac myosin-binding protein-C (cMyBP-C; 20\%) on chromosome 14 and 11, respectively [9]. The inheritance of these mutations is autosomal dominant with age-related penetrance, although autosomal recessive mutations have also been described [10]. In about $5 \%$ of families, there are at least 2 distinct HCM causing mutations present [11]. Homozygous, double or compound heterozygous mutations usually trigger more severe symptoms, including sudden cardiac death (SCD) [5].

In the absence of loading conditions (eg. hypertension, aortic stenosis) or a known systemic disease (eg. Fabry-disease, amyloidosis), HCM can be defined by the presence of abnormal wall thickness $(\geq 15$ $\mathrm{mm}$ ) in adults [11]. However, genetic testing is more and more frequently used for corroborating this diagnosis. Here, we describe a case where after the diagnosis of HCM with conventional methods using our new NGS-based testing panel clinically valuable additional information became available which helped to diagnose and map HCM in a family, and also assisted decision making on the implantation of a cardioverter defibrillator (ICD). 
Citation: Horvath VJ, Arvai K, Kosa JP, Balla B, Tobias B, et al. (2017) Next-generation Sequencing in the Clinical Decision Making in Hypertrophic Cardiomyopathy. Next Generat Sequenc \& Applic 4: 145. doi:10.4172/2469-9853.1000145

Page 2 of 4

\section{Materials and methods}

\section{Clinical diagnosis}

Briefly, after successful reanimation, a 7 years old girl was diagnosed with HCM. Cardiac MRI was performed on the patient's two older sisters ( 9 and 10 years old) and in both cases signs of HCM were confirmed. Representative MRI images are shown in Figure 1. The sudden cardiac death together with the diagnosed HCM indicated the implantation of ICD in the 7 years old patient. However, considering the predominantly autosomal dominant inheritance of HCM, the genetic nature of the family members was investigated. This genetic test panel developed in the 1st Department of Medicine, Semmelweis University.

\section{DNA isolation}

Genomic DNA was isolated from $200 \mu \mathrm{l}$ of peripheral blood using Reliaprep Blood gDNA Miniprep System (Promega, Madison, WI). Briefly, the blood samples were digested with Proteinase K solution in the presence of Cell Lysis Buffer, and after $10 \mathrm{~min}$ of incubation at $56^{\circ} \mathrm{C}$, DNA was bound to ReliaPrepTM Binding Column. After three washes, DNA was eluted into $50 \mu \mathrm{l}$ of nuclease-free water. The concentration of the isolated DNA was determined with Qubit dsDNA HS Assay Kit (Life Technologies, Carlsbad, CA).

\section{Library preparation and next-generation sequencing}

For sequence capture, SureSelectXT Human All Exon V4 Kit (Agilent) was used, according to the manufacturer's instructions (http://www.chem.agilent.com/library/usermanuals/Public/ G753090000_SureSelect_IlluminaXTMultiplexed_1.7.pdf). Briefly, in the first step, gDNA was sheared into small fragments using mechanical shearing procedure, then sequencing adaptors and indices were ligated to the small DNA fragments, during the library preparation. The sample was hybridized with $120 \mathrm{mer}$ biotinylated RNA library baits for the highest specificity. DNA fragments with hybridized probes were selected using magnetic streptavidin beads and after PCR amplification was loaded into an Illumina HiSeq 2500 sequencer.

\section{Mutation validation}

The PCR primers were designed using Primer3Plus (http:// primer3plus.com/) software. Roche FastStart TaqMan Probe Master (Roche) kit was used to amplify the target regions and the PCR program was as follows: $95^{\circ} \mathrm{C} 10 \mathrm{~min}, 40$ cycles of $95^{\circ} \mathrm{C} 30 \mathrm{~s}, 60^{\circ} \mathrm{C} 30$ $\mathrm{s}, 72^{\circ} \mathrm{C} 45 \mathrm{~s}$ and the final step was $72^{\circ} \mathrm{C} 5 \mathrm{~min}$. PCR products were enzymatically cleaned using ExoSAP IT (Affymetrix, Santa Clara, CA) according to the manufacturer's instructions. Sanger sequencing was performed using BigDye Terminator v3.1 Cycle Sequencing Kit (Life Technologies) using an ABI 3130 instrument (Life Technologies).

\section{Data analysis}

Data from the sequencing run was analyzed using the platformspecific pipeline software Illumina RTA and CASAVA (1.8.2) for basecalling, trim adapter and primer sequences, filtering out poor quality reads, and de-multiplex the reads according to the barcode sequences. BWA 0.5.9-r16 algorithm (http://bio-bwa.sourceforge.net/) was used to align the reads to the hg19 human reference genome, then Samtools (http://samtools.sourceforge.net/) was selected to run to search for germline variants in the targeted regions. Integrative Genomics Viewer was used for visualization of the mapped reads $\left({ }^{*}\right)$. Variants were reviewed and annotated using ANNOVAR 2012/06/21 (http:// www.openbioinformatics.org/annovar/), dbSNP(http://

www.ncbi.nlm.nih.gov/projects/SNP/) and 1000 Genome database (http://www.1000genomes.org/).

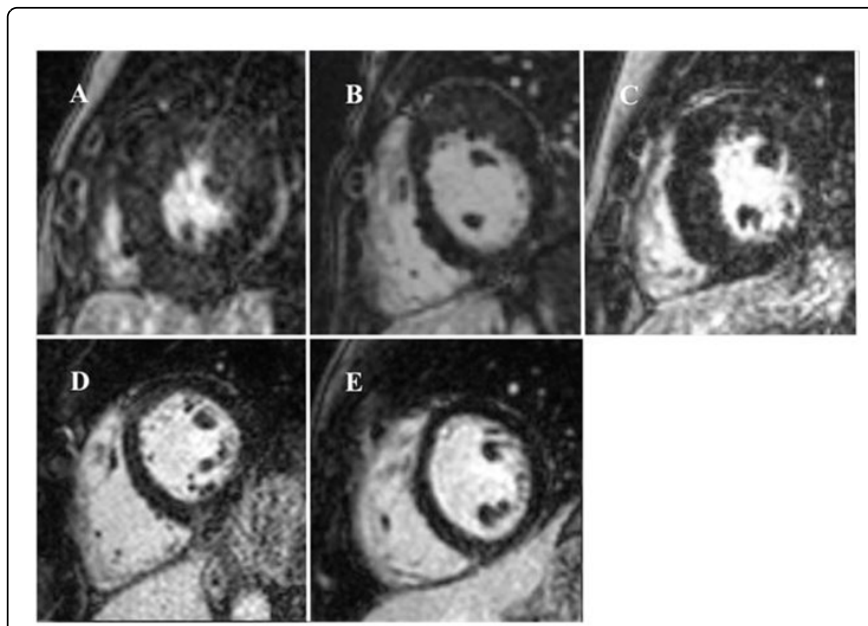

Figure 1: Representative short axis LGE MRI images in the papillary muscle level of the family members' hearts. The most severe, even hypertrophic cardiomyopathy can be detected in the panel A (reanimated girl). Also, severe lesions are present in the heart of the 9 and 10 years old (panel B and C, respectively) sisters but the hypertrophy is asymmetric, localized rather in the septal regions. All the three children carried the MyBPC mutations in compound heterozygous form. Signs of mild hypertrophy was detected in the father's heart (panel D), who carried only the point mutation. No major signs of disease could be see seen in the mother's heart (panel E), although she had a frameshift mutation which severely destroys the structure of MyBPC protein.

For variant interpretation, Ingenuity Variant Analysis Pipeline (Ingenuity, Rewood City, CA) and HGMD Professional 2014.1 (http:// www.hgmd.org/) were also used. Pathogenic status of the variant was stated if it was a missense variant with $<1 \%$ minor allele frequency and/or the variant was listed in the literature or in the databases as a pathogenic alteration. The following genes were investigated for genetic variation: MYBPC3; TNNI3; TNNT2; MYH7; TTN; LMNA, MYH6; SCN5A; KCNQ1; KCNH2; SCN4B; KCNE1; KCNE2; KCNJ2; CACNA1C; CASQ2; hRYR2; DSP; PKP2; CAV3; ACE; AGTR1; CMA1; AGT1; CYP11B2; GPD1L; KCNE3; SCN3B; SCN4B; SNTA1; AKAP9; ANK2; CACNB2; SCN1B; TPMI1; DSG2; DSC2; KCNJ5; MYLK2; MYL2; MYL3; MYH6; CMH4; PRKAG2; ACTC1; TNNC1; PLN.

Called and deleterious variants were confirmed by Sangersequencing. The Sanger sequence data were investigated using ABI Sequence Scanner 1.0 (Life Technologies) and BioEdit (http:// www.mbio.ncsu.edu/bioedit/bioedit.html).

\section{Results}

Altogether, 78,987,512 sequencing reads were generated, resulting in an average coverage of targeted regions at $96,2 \mathrm{X}$. Non-redundant unique reads (uniquely mapped to human genome) number was 
$74,770,198$. The on-target read number was 57,092,646 (76.4\%), yielding $97.8 \% 10 \mathrm{X}$ coverage of the targeted regions. As a result, 341 variants were called in the selected cardiomyopathy-related genes.

We have identified two variants in the TTN gene with unknown clinical significance (rs201043950 and rs72646808), and also identified two disease-causing heterozygous mutations in the MYBPC3 gene (p.R495Q and p.S593fs ${ }^{\star} 11$ ). The p.R495Q mutation is not present in Exome Variant Server database (http://evs.gs.washington.edu/EVS/), while the ExAc database (http://exac.broadinstitute.org/) contains it with a reported frequency of 0.000008294 . After the disease-causing mutations were found, all of the family members were screened, targeting these two mutations. The mother carried the frameshift mutation (p.S593fs* ${ }^{*} 1$ ) while the father's genome contained the point mutation (p.R495Q). All the children were compound heterozygous.

Our approach revealed two different mutations present in the family. The parents carried the mutations separately while all the 3 children possessed both mutations in a compound heterozygous form. The detailed analysis of clinical data did not show a mutation-specific sign, however the sudden death of the younger sister itself is a IIb/C indication of ICD implantation for the 2 older sisters [12]. The decision of ICD implantation was made even more difficult by the fact that the implantation in children may be associated with a $25 \%$ rate of complications. However, considering the clinical data together with the severe genetic alterations, finally, implantation of ICD was decided into both sisters as primary prevention. Later, the father also received an ICD after a $30 \mathrm{~s}$ collapse with unknown origin. Since the mother does not have severe morphological or clinical sign of HCM, ICD implantation was not indicated in her.

\section{Discussion}

Routine whole exome-sequencing (WES) was selected for this study instead of targeted gene panel sequencing in order to cover all potential genetic factors causing cardiovascular diseases. Our goal was to avoid the long list of variants which are unrelated to the symptoms of the patient, and that can slow down the analysis and interpretation. Because of this, only a fragment of the generated data was analyzed, searching for disease-causing mutations in known cardiomyopathyassociated genes compiled by us. Thus, we have established an NGSbased method that provides a fast and cost-effective approach to set up the genetic basis of the diagnosis.

With this approach we found 2 disease causing mutations present in the family members. The frameshift mutation (p.S593fs*11) was present in the mother and in all the 3 sisters where the mutation was detected together with the known missense mutation (p.R495Q; cgg $>$ cag) in compound heterozygous formation. The compound heterozygous inheritance of the two mutations in all the three sisters is an interesting phenomenon but can be explained rather with accidental than controlled physiological mechanisms. The frameshift mutation changes the basic structure of human cMyBPC protein. In healthy subjects, the molecule contains 1274 amino acids and its main task is to modulate contractility responses for beta-adrenergic stimulation. Its $\mathrm{C}$-terminal-anchoring-domain (C10) binds strongly to light meromyosin while $\mathrm{C} 8-10$ domains interact with titin [13]. The $\mathrm{N}$ terminal part of the molecule contains the actin binding region. The newly diagnosed frameshift mutation (p.593fs* 11$)$ is located in exon 17 , and causes an early termination in translation; the truncated protein will contain only 602 amino acids. This shorter protein shares the same amino acid sequence with the original one until the 592th amino acid, and between the 593-602 positions a PHRAGPQTDH sequence is formed, then, translation is terminated. Taken together, the mutation forming allele will provide a much shorter protein with $\mathrm{C}$ terminal fragment lost. A mutation in similar location has already been published (K600fs); with this mutation, atrial fibrillation was diagnosed at young age but no any other specific sign or clinical symptoms including sudden cardiac death was observed [14].

The known point mutation (p.R495Q) is carried alone only by the father and together with the p. $593 \mathrm{fs}^{\star} 11$ in the sisters. In the father, the mutation caused only moderate changes in the cardiac phenotype. However, recently a collapse with unknown origin also occurred to the father, which justified an ICD implantation. Based on the literature, this mutation has not been linked to a specific clinical sign. Also, other specific type of cMyBPC mutations do not present with statistically significant difference in clinical phenotype [15].

Obvious causes like unknown mutations or escape mechanisms, or epigenetic differences could explain the different phenotypes with the same known mutations among the sisters. However, no truncated cMyBPC proteins can be detected in myocardial biopsy samples from patients diagnosed with HCM. Nevertheless, a reduction in the amount of full-length cMyBPC protein has been noted [16]. A possible mechanism that can partly explain this phenomena is that mutant cMyBPC mRNA or proteins can undergo a rapid degradation by the ubiquitin-proteosome system and in this way the incorporation of mutant proteins into the sarcomere can be prevented [17]. Moreover, the mechanism of nonsense mediated mRNA decay, which occur on mRNAs that contain a stop codon upstream of the last exon-exon junction of the gene, will eliminate the MYBPC 3 mRNA containing the frameshift mutation. This mechanism also decreases the protein level of cMyBPC. This degradation process and the consequent missing of their incorporation to the tissue may also be an explanation of the similarity among the phenotypic appearance of the different mutations.

The selection of patients for ICD implantation is also a matter of debate. In our case, the youngest sister received the device after SCD as secondary prevention. However, the two other sisters and the father underwent this procedure as a primary prevention. The 2011 ACC/AHA guideline recommended the ICD implantation in adults for primary prevention in a class IIa indication [18]. However, the 2014 ESC guideline recommends ICD implantation with IIa indication if the 5 year mortality risk is higher than $6 \%$; between $4-6 \%$ risk the indication is IIb [12]. The conventional risk factors (like maximum left ventricular thickness, left atrial diameters, maximal LVOT gradient, etc.) sometimes do not have enough predictive value. Recently, late gadolinium enhancement in MRI (LGE-MRI) for the detection of myocardial fibrosis was reported as a potential risk factor to predict SCD [19]. However, a detailed analysis of the double or compound heterozygous mutations revealed that even in the absence of conventional risk factors, genetic testing also can predispose to adverse disease progression and multiple sarcomere mutations are associated with a risk of sudden death [20].

In summary, with the help of our NGS-based method, we found a recently described mutation in $c M y B P C$ gene which obviously causes functional loss of the encoded protein but does not come with morphological change in the heart. Also, we mapped the sarcomere mutations in a family with known HCM after the sudden cardiac death of the youngest child. The frameshift mutation was present in the three children together with the point mutation in compound heterozygous form. Together with the available clinical approaches, this genetic test 
Citation: Horvath VJ, Arvai K, Kosa JP, Balla B, Tobias B, et al. (2017) Next-generation Sequencing in the Clinical Decision Making in Hypertrophic Cardiomyopathy. Next Generat Sequenc \& Applic 4: 145. doi:10.4172/2469-9853.1000145

Page 4 of 4

is an additional step to estimate the potential risk of SCD and could help to choose the optimal treatment. This case further reveals the importance of the available genetic tests in therapeutic decisions.

\section{References}

1. Von Bubnoff A (2008) Next-generation sequencing: the race is on. Cell 132: 721-723.

2. Sanger F, Nicklen S, Coulson AR (1977) DNA sequencing with chain terminating inhibitors. Proc Natl Acad Sci USA 74: 5463-5467.

3. Árvai K, Horváth $\mathrm{P}$, Balla B, Tobiás B, Kató K, et al. (2016) Nextgeneration sequencing of common osteogenesis imperfecta-related genes in clinical practice. Sci Rep 6: 28417.

4. Rothberg JM, Hinz W, Rearick TM, Schultz J, Mileski W, et al. (2011) An integrated semiconductor device enabling non-optical genome sequencing. Nature 475: 348-352.

5. Kelly M, Semsarian C (2009) Multiple mutations in genetic cardiovascular disease: A marker of diasease severity? Circ Cardiovasc Genet 2: 182-190.

6. Maron BJ, Gardin JM, Flcak JM, Gidding SS, Kurosaki TT, et al. (1995) Prevalence of hypertrophic cardiomyopathy in a general population of young adults. Echocardiographic analysis of 4111 subjects in the CARDIA study. Coronary artery risk development in (Young) adults. Circulation 92: 785-789.

7. Nugent AW, Daubeney PE, Chondros P, Carlin JB, Colan SD, et al. (2005) Clinical features and outcomes of childhood hypertrophic cardiomyopathy: results from a national population-based study. Circulation 112: 1332-1338.

8. Lind JM, Chiu C, Semsarian C (2006) Genetic basis of hypertrophic cardiomyopathy. Expert Rev Cardiovasc Ther 4: 927-934.

9. Maron BJ, Maron MS (2013) Hypertrophic cardiomyopathy. Lancet 381: 242-255.

10. Olson TM, Karst ML, Whitby FG, Driscoll DJ (2002) Myosin light chain mutation causes autosomal recessive cardiomyopathy with mid-cavitary hypertrophy and restrictive physiology. Circulation 105: 2337-2340.

11. Ingles J, Doolan A, Chiu C, Seidman J, Seidman, et al. (2005) Compound and double mutations in patients with hypertrophyc cardiomyopathy: implications for genetic testing and counselling. J Med Genet 42: e59.
12. Authors/Task Force members, Elliott PM, Anastasakis A, Borger MA, Borggrefe M, et al. (2014) 2014 ESC Guidelines on diagnosis and management of hypertrophic cardiomyopathy: the Task Force for the Diagnosis and Management of Hypertrophic Cardiomyopathy of the European Society of Cardiology (ESC). Eur Heart J 35: 2733-2779.

13. Flashman E, Redwood C, Moolman-Smook J, Watkins H (2004) Cardiac myosin binding protein C: its role in physiology and disease. Circ Res 94 1279-1289.

14. Rodríguez-García M, Monserrat L, Ortiz M, Fernández X, Cazón L, et al. (2010) Screening mutations in myosin binding protein C3 gene in a cohort of patients with hypertrophic cardiomyopathy. BMC Med Genet 11: 67-76.

15. Van Driest SL, Vasile VC, Ommen SR, Will ML, Tajik AJ, et al. (2004) Myosin binding protein $\mathrm{C}$ mutations and compound heterozygosity in hypertrophic cardiomyopathy. J Am Coll Cardiol 44: 1903-1910.

16. van Dijk SJ, Dooijes D, dos Remedios C, Michels M, Lamers JM, et al. (2009) Cardiac myosin-binding protein C mutations and hypertrophic cardiomyopathy: haploinsufficiency, deranged phosphorylation, and cardiomyocyte dysfunction. Circulation 119: 1473-1483.

17. Schlossarek S, Mearini G, Carrier L (2011) Cardiac myosin-binding protein $\mathrm{C}$ in hypertrophic cardiomyopathy: mechanisms and therapeutic opportunities. J Mol Cell Cardiol 50: 613-620.

18. Gersh BJ, Maron BJ, Bonow RO, Dearani JA, Fifer MA, et al. (2011) 2011 ACC/AHA guideline for the diagnosis and treatment of hypertrophic cardiomyopathy: executive summary: a report of the American College of Cardiology Foundation/American heart association task force on practice guidelines. Circulation 124: 2761-2796.

19. Green JJ, Berger JS, Kramer CM, Salerno M (2012) Prognostic value of late gadolinium enhancement in clinical outcomes for hypertrophic cardiomyopathy. JACC Cardiovasc Imaging 5: 370-377.

20. Maron BJ, Maron MS, Semsarian C (2012) Double or compound sarcomere mutations in hypertrophic cardiomyopathy: A potential link to sudden death in the absence of conventional risk factors. Heart Rhythm 9: 57-63. 